\title{
Phase chaos in coupled oscillators
}

\author{
Oleksandr V. Popovych, ${ }^{1}$ Yuri L. Maistrenko, ${ }^{1,2,3}$ and Peter A. Tass ${ }^{1,4}$ \\ ${ }^{1}$ Institute of Medicine and Virtual Institute of Neuromodulation, Research Centre Jülich, 52425 Jülich, Germany \\ ${ }^{2}$ Central Institute for Electronics, Research Center Jülich, 52425 Jülich, Germany \\ ${ }^{3}$ Institute of Mathematics, National Academy of Sciences of Ukraine, 01601 Kyiv, Ukraine \\ ${ }^{4}$ Department of Stereotaxic and Functional Neurosurgery, University Hospital, 50924 Cologne, Germany
}

(Received 1 December 2004; published 6 June 2005)

\begin{abstract}
A complex high-dimensional chaotic behavior, phase chaos, is found in the finite-dimensional Kuramoto model of coupled phase oscillators. This type of chaos is characterized by half of the spectrum of Lyapunov exponents being positive and the Lyapunov dimension equaling almost the total system dimension. Intriguingly, the strongest phase chaos occurs for intermediate-size ensembles. Phase chaos is a common property of networks of oscillators of very different natures, such as phase oscillators, limit-cycle oscillators, and chaotic oscillators, e.g., Rössler systems.
\end{abstract}

DOI: 10.1103/PhysRevE.71.065201

PACS number(s): 05.45.Xt, 05.45.Jn

The Kuramoto model of coupled phase oscillators [1],

$$
\dot{\psi}_{i}=\omega_{i}+\frac{K}{N} \sum_{j=1}^{N} \sin \left(\psi_{j}-\psi_{i}\right), \quad i=1, \ldots, N,
$$

is one of the most popular and often cited networks in modern nonlinear science and application [2]. It reflects generic properties of ensembles of globally coupled limit-cycles oscillators of very different nature. Its applications cover a wide range of self-organizing systems in the natural sciences and medicine, e.g., Josephson-junction arrays [3], semiconductor lasers arrays [4], coupled chemical reactions [5], and cardiac pacemaker cells [6]. Several neurological diseases, such as Parkinson's disease, are characterized by a synchronization of ensembles of oscillatory neurons in particular brain areas, where the normal firing is supposed to be uncorrelated [7]. The development of desynchronizing deep brain stimulation for the therapy of such diseases [8], together with the successful clinical tests of this therapeutic approach [9], have led to an increasing interest in nonsynchronized states of networks of oscillators. The design of optimal techniques for selective disruption of synchronization requires sufficient knowledge of relevant features of the variety of possible desynchronized states.

The Kuramoto model (1) exhibits incoherent behavior at small and intermediate values of the coupling coefficient $K$, and a spontaneous transition to collective synchronization as $K$ exceeds a certain threshold $K_{c}$. In spite of numerous studies during the last two decades, being mainly based on a statistical approach in the thermodynamic limit $N \rightarrow \infty$, the finite-dimensional character of model (1) is still far from being understood $[2,10]$. In particular, unsolved and challenging problems refer to global stability and convergence.

In this paper, we present a complex high-dimensional chaotic regime in the Kuramoto model (1), as a typical phenomenon of phase chaos [11] in coupled oscillator systems. We find that phase chaos in coupled oscillators extends over small and intermediate coupling strength up to the synchronization transition. This type of chaos arises as soon as $N=4$ or more oscillators interact. With increasing system size
$N$, its complexity develops and phase chaos becomes high dimensional: The number of positive Lyapunov exponents (LE) is found to equal $(N-2) / 2(N$ even $)$ or $(N-3) / 2(N$ odd), and the Lyapunov dimension reaches almost the total system dimension $N$. We also show that the intensity of phase chaos in coupled oscillators, as given by the maximal LE, decays quadratically with coupling strength $K$ and inverse proportionally to ensemble size $N$. Intriguingly, the "most chaotic" phase chaos emerges for intermediate-size ensembles.

Phase chaos in coupled oscillators is a robust phenomenon. We found phase chaos in the Kuramoto model (1) for both uniform and Gaussian distributions of the natural frequencies $\omega_{i}$. This type of chaos is generated by intrinsic phase interactions, and is also typical for other ensembles of oscillators of very different nature. We demonstrate that phase chaos emerges in networks of limit-cycle oscillators and coupled chaotic Rössler systems. In the case of coupled chaotic oscillators, phase chaos manifests itself in the appearance of additional chaotic components, where additional positive LEs (with respect to those of individual oscillators) emerge.

First, we consider the simplest coupled oscillator system, where phase chaos occurs: $N=4$ in Eq. (1). By introducing the phase difference variables $\varphi_{i}=\psi_{i+1}-\psi_{i}, i=1,2,3$, the dimension of the Kuramoto model is reduced by one. The system dynamics is then governed by a three-dimensional torus flow $\Phi$. In Fig. 1(a) three LEs of the flow $\Phi$ are plotted versus the coupling strength $K$. The maximal LE $\lambda_{1}$ is positive for values of $K$ between $K_{0} \approx 0.94$ and $K_{1} \approx 1.22$, which implies the existence of a chaotic attractor $A$ [see Fig. 1(c)].

Before the chaotic attractor $A$ is born, i.e., for $K<K_{0}$, the system dynamics is mostly quasiperiodic [solid torus curve in Fig. 1(b)]. The transition to chaos at $K_{0}$ ensues in accordance with the torus destruction scenario [16]. With increasing $K$, first, the dynamics fits into a resonance [window for $K \in\left(K_{s n}, K_{0}\right)$, see inset in Fig. 1(a)]: A stable periodic orbit $P$ [crosses in Fig. 1(b)] and a saddle $Q$ of the resonance 9: 10 appear in a saddle-node bifurcation at $K=K_{s n}$. Then, at the exit from the resonance, the chaotic attractor $A$ is born [black 

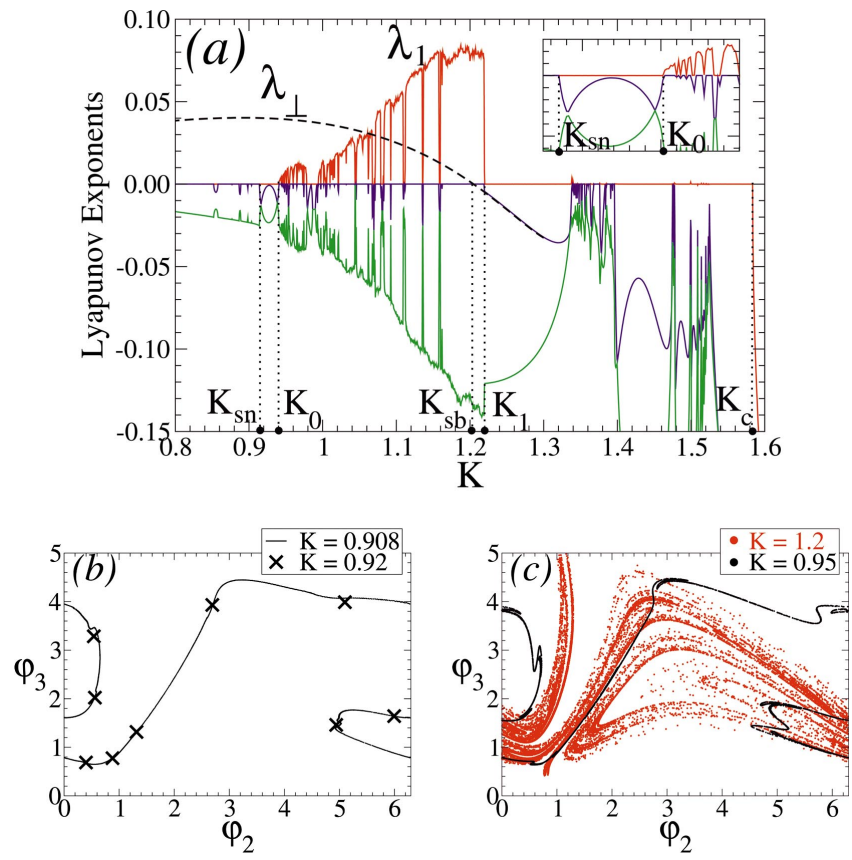

FIG. 1. (Color) Chaos in the Kuramoto model (1) of $N=4$ phase oscillators. Natural frequencies $\omega_{i}=-1+2(i-1) / 3, i=\overline{1,4}$. (a) Lyapunov exponents vs parameter $K$, with enlargement in the inset for $K \in[0.91 ; 0.96] . \lambda_{\perp}$ is a transverse LE of the invariant manifold $\mathcal{M}=\left\{\varphi_{1}=\varphi_{3}\right\}$; (b) regular, and (c) chaotic attractors in the Poincare section $\varphi_{1}=0$.

dots in Fig. 1(c)]. With a further increase of $K$, the fractal structure of $A$ becomes clearly visible [red dots in Fig. 1(c)]. Finally, the chaotic attractor $A$ is destroyed in a boundary crisis at $K=K_{1}$. Analogous chaotic phase dynamics with one positive LE is found in the Kuramoto model (1) of dimension $N=5$. Phase chaos becomes more developed for $N=6$ and $N=7$, where two LEs are positive.

To shed light on the LEs' arrangement for larger $N$, consider an example with $N=20$ Kuramoto phase oscillators. The fine structure of all LEs in this case is shown in Fig. 2(a). Our calculations reveal that for the values of $K$ smaller than $K_{1} \approx 0.55$, nine LEs are positive $\left(\lambda_{1}>\lambda_{2}>\cdots\right.$ $>\lambda_{9}>0$ ), and nine LEs are negative. As $K$ increases from zero, the magnitudes of LEs, both positive and negative, grow quadratically (see also Ref. [18])

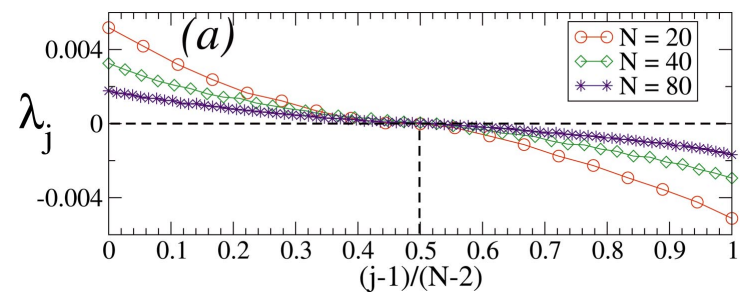

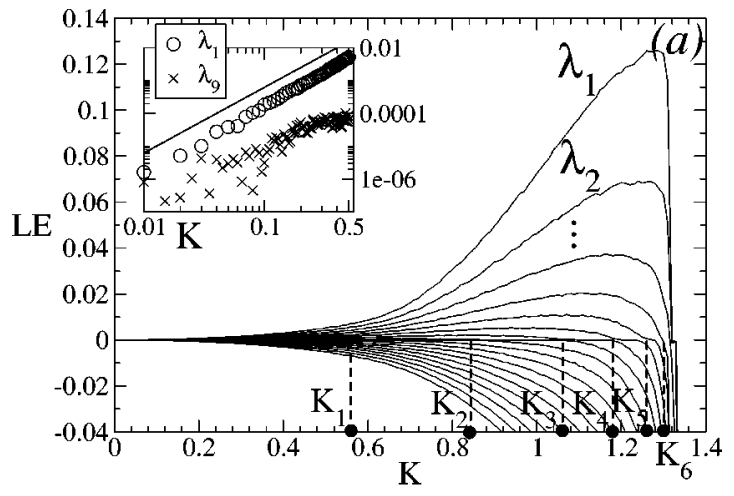

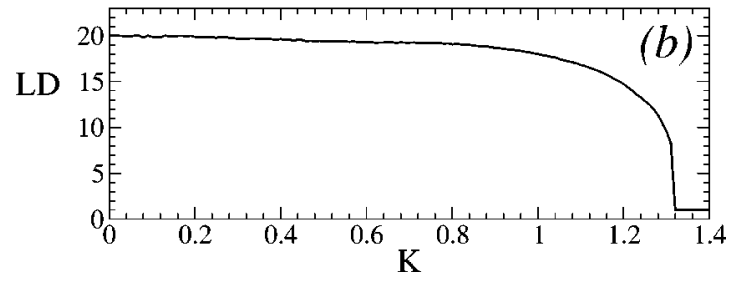

FIG. 2. High-dimensional chaos in the Kuramoto model (1) of $N=20$ phase oscillators. Natural frequencies $\omega_{i}=-1+2(i-1) / 19$, $i=\overline{1,20}$. (a) The nine largest Lyapunov exponents are positive for the coupling parameter range $0<K<K_{1}$ and successively change their sign to negative at $K=K_{i}, i=1, \ldots, 9$. In the inset two exponents $\lambda_{1}$ and $\lambda_{9}$ are shown in a log-log scale. The straight line there is obtained by direct fit of the values of $\lambda_{1}$ and has slope of 2.05. (b) The Lyapunov dimension.

$$
\lambda_{i} \sim K^{2}, \quad \text { as } K \rightarrow 0,
$$

which is supported in the inset in Fig. 2(a) by graphs of $\lambda_{1}$ and $\lambda_{9}$ in the log-log scale. The chaotic behavior is fully developed for $K<K_{1}$ : The Lyapunov dimension [17] almost equals the total system dimension $N$. This is illustrated in Fig. 2(b). As $K$ increases, the Lyapunov dimension decays, and the positive LEs change their sign to negative, one after another, at some coupling parameter points $K_{1}<K_{2}<\cdots$ $<K_{9}$ as depicted in Fig. 2(a). Finally, at $K=K_{9}$, the maximal LE $\lambda_{1}$ drops down through zero and the dynamics becomes periodic up to the synchronization transition at $K=K_{c}$. However, the extent of chaos, which is given by the magnitude of the maximal LE $\lambda_{1}$, grows with increasing $K$ from zero. It reaches its maximum at some coupling parameter value $K_{\max }$, then rapidly falls and vanishes at $K_{c h}=K_{9}$.

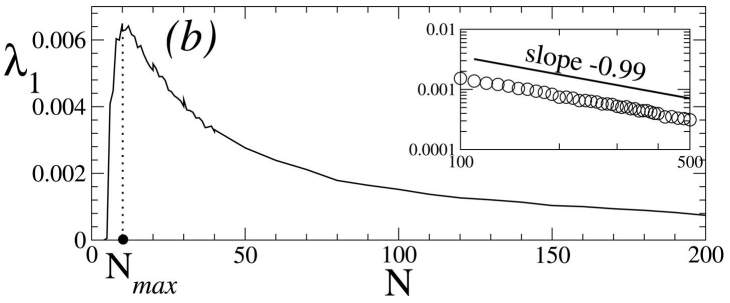

FIG. 3. (Color) (a) Lyapunov spectrum $\left\{\lambda_{j}\right\}$ of the Kuramoto model (1) vs the normalized number of exponents for ensemble sizes $N=20$ (red circles), 40 (green diamonds), and 80 (blue asterisks). (b) The maximal LE $\lambda_{1}$ vs ensemble size $N$. The maximum is attained $N_{\max }=10$. The inset shows $\lambda_{1}$ (circles) in the log-log scale and a direct fit (3) of the values of $\lambda_{1}$ with slope -0.99 (straight line). Parameters $K=0.5$ and $\omega_{i}=-1+2(i-1) /(N-1), i=\overline{1, N}$. 


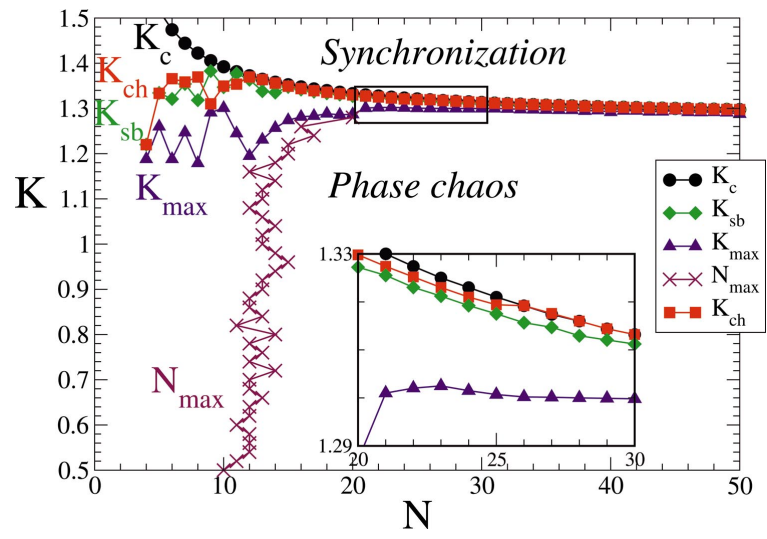

FIG. 4. (Color) Phase diagram of the Kuramoto model (1). Natural frequencies $\omega_{i}=-1+2(i-1) /(N-1), i=\overline{1, N}$. Bifurcation curves are shown: $K_{c}$ (black circles)—synchronization transition; $K_{c h}$ (red squares) - transition to chaos; $K_{s b}$ (green diamonds)symmetry breaking. Also, $K_{\max }$ (blue triangles) and $N_{\max }$ (maroon crosses) indicate parameter values, where $\max _{K} \lambda_{1}$ and $\max _{N} \lambda_{1}$ are attained, respectively. An enlargement of small rectangle is given in the inset.

In Fig. 3(a) the LEs are plotted in numerical order for ensemble sizes $N=20$ (circles), $N=40$ (diamonds), and $N$ $=80$ (asterisks) for $K=0.5$. The points of the Lyapunov spectrum clearly fit, more and more densely, a smooth curve that converges to the zero axis as $N \rightarrow \infty$ or $K \rightarrow 0$. In Fig. 3(b) the value of the maximal LE $\lambda_{1}$ is plotted versus the number of oscillators $N . \lambda_{1}$ becomes positive at $N=4$. Thereupon, it grows with increasing $N$ and reaches its maximal value $\max _{N} \lambda_{1}(N)$ at some $N_{\max }$. By increasing $N$ beyond $N_{\max }$, $\lambda_{1}$ decreases and vanishes as $N \rightarrow \infty$. Our numerical approximations provide the decay rate of $\lambda_{1}$ as inversely proportional to $N$

$$
\lambda_{1} \sim N^{-1}, \quad \text { as } N \rightarrow \infty .
$$

Legitimacy of the scaling law (3) can also be supported by analytical arguments [19].

Let us consider the Kuramoto model (1) with the natural frequencies $\omega_{i}$ evenly spaced in the interval $[-\gamma, \gamma]$, $\omega_{i}=-\gamma+2 \gamma(i-1) /(N-1), \quad i=1, \ldots, N$, corresponding to a uniform density $g(\omega)=1 /(2 \gamma)$ for $|\omega|<\gamma$ and 0 otherwise. Without loss of generality, one can put $\gamma=1$. Indeed, by choosing a new time $t \mapsto t^{\prime}=\gamma t$ one obtains $\psi_{i}(t ; \gamma ; K)$ $=\psi_{i}(\gamma t ; 1 ; K / \gamma)$. This allows us to conclude that the LEs depend on $\gamma$ as follows: $\lambda_{i}(\gamma, K)=\gamma \lambda_{i}(1, K / \gamma)$. With Eq. (2) this implies that

$$
\lambda_{i} \sim K^{2} / \gamma, \quad \text { as } \gamma \rightarrow \infty .
$$

Therefore, if $K$ and $N$ are fixed, LEs vanish inversely proportional to $\gamma$. On the other hand, $\max _{K}\left(\lambda_{i}\right)$ linearly grows proportionally to $\gamma$.

In Fig. 4 the regions of two qualitatively different types of long-term behavior in the Kuramoto model (1) are delineated in the $(N, K)$-parameter plane for $\gamma=1$. Synchronization: Above the black curve $K=K_{c}$ there is only the phase-locked dynamics given by a unique stable equilibrium of the re- duced system of the phase differences. Phase chaos: Below the red curve $K=K_{c h}$ at least one LE is positive, and the dynamics is chaotic.

For an essential part of the parameter diagram in Fig. 4, the system dynamics is restricted to a symmetric invariant manifold $\mathcal{M}=\left\{\varphi_{i}=\varphi_{N-i}\right\}, \quad \varphi_{i}=\psi_{i+1}-\psi_{i}, \quad$ of dimension $N_{0}=[N / 2]$. The manifold $\mathcal{M}$ exists provided the natural frequencies are symmetrically allocated around the mean frequency $\Omega$ : $\omega_{i+1}-\omega_{i}=\omega_{N-i+1}-\omega_{N-i}$. In-manifold dynamics is stable in the whole phase space provided all LEs transverse to the manifold are negative [see Fig. 1(a) for $N=4$ ]. As shown in Fig. 4, this is valid for values of $N$ and $K$ above the symmetry breaking bifurcation curve $K=K_{s b}$. Note that the symmetry breaking bifurcation curve $K=K_{s b}$ lies below the synchronization onset curve $K=K_{c}$. Therefore, the desynchronization transition in the Kuramoto model (1) takes place inside the manifold $\mathcal{M}$.

We find that the in-manifold dynamics is given by a Winfree model [20]. For even $N$ it takes the form

$$
\dot{\psi}_{i}=\omega_{i}-\frac{K}{N_{0}} \sin \psi_{i} \sum_{j=1}^{N_{0}} \cos \psi_{j}, \quad i=1, \ldots, N_{0} .
$$

(For odd $N$ the model is similar [21].) The reduction to the Winfree model is valid for all $K>K_{s b}$ and allows us to find the bifurcation moment $K_{c}$ analytically for any $N$ in Eq. (1) [21]. In the thermodynamic limit $N \rightarrow \infty, K_{c}$ converges to Kuramoto's critical bifurcation value $K_{c, \infty}=4 / \pi$ [1], and the convergence scales as $1 / N$.

With an increase of $N$, not only the synchronization onset curve $K=K_{c}$, but also three more bifurcation curves, $K=K_{c h}$, $K=K_{s b}$, and $K=K_{\max }$, converge to $K_{c, \infty}=\pi / 4$ being ordered as $K_{\max }<K_{s b}<K_{c h} \leqslant K_{c}$ (Fig. 4). It is worth nothing that $K_{c h}$ coincides with $K_{c}$ for all $N \geqslant 26$ (see inset in Fig. 4), and then chaos emerges in the Kuramoto model just at the desynchronization transition. Furthermore, the distance between $K_{\max }$ and $K_{c}$ quickly diminishes as $N$ increases, which implies that for large $N$ the maximal level of chaoticity is achieved almost immediately after the desynchronization. A typical shape of the maximal LE $\lambda_{1}$ as a function of $K$ is shown in Fig. 2(a), where $N=20$.

Finally, the bifurcation curve $N=N_{\max }$ depicted in Fig. 4 indicates the parameter values, where $\lambda_{1}$ attains its maximum as a function of $N$. For a given coupling strength $K, N_{\max }$ tells us how many phase oscillators have to be coupled in the Kuramoto model (1), to achieve a maximal level of chaos. Intriguingly, the maximally chaotic ensemble size $N_{\max }$ mostly lies between 10 and 15 . We conclude that intermediate-size groups of oscillators can generate much more intensive phase chaos than small or big ones. A typical dependence of $\lambda_{1}$ on ensemble size $N$ is shown in Fig. 3(b), where $K=0.5$.

Phase chaos is an essential property of coupled oscillator systems and it is by no means restricted to the Kuramoto model (1). Rather, it also occurs in globally coupled limitcycle oscillators and Rössler systems, as we demonstrate in Fig. 5. Also, phase chaos has been detected in terms of positive LEs in previous studies of globally coupled Ginzburg- 

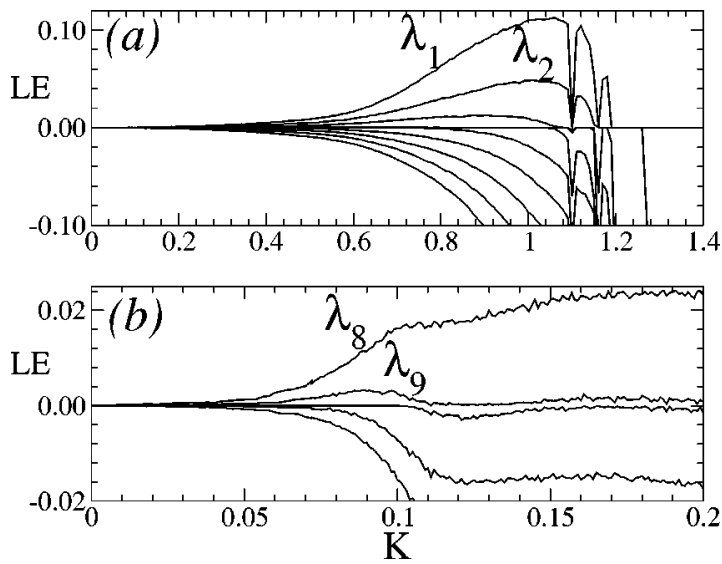

FIG. 5. (a) Ten largest LEs $\lambda_{1}, \ldots, \lambda_{10}$ for 10 globally coupled limit-cycle oscillators $\dot{Z}_{j}=\left(a_{j}+i \omega_{j}-\left|Z_{j}\right|^{2}\right) Z_{j}$ with $a_{j}=1$ and $\omega_{j}$ uniformly distributed in the interval $[-1 ; 1]$. (b) Five LEs $\lambda_{8}, \ldots, \lambda_{12}$ for seven globally coupled chaotic Rössler oscillators with parameters as in Ref. [22] and with $\omega_{j}$ uniformly distributed in the interval $[0.95 ; 1.05]$.

Landau-type oscillators [23], phase oscillators with nearestneighbor coupling [24], and coupled Lorenz systems [18].

In summary, we have discovered a phenomenon of highdimensional phase chaos in the Kuramoto model (1) and other ensembles of coupled oscillators. Nonlinear interac- tions cause chaoticity of the collective phase dynamics although individual oscillators behave only periodically when left uncoupled. We have shown that the phase chaos is characterized by $(N-2) / 2(N$ even) or $(N-3) / 2(N$ odd $)$ positive Lyapunov exponents, and that the Lyapunov dimension reaches almost the total system size $N$. Moreover, the Lyapunov exponents are scaled quadratically with $K$, as $K$ vanishes, and inverse proportionally to $N$, as $N$ grows. The results are carried out for the Kuramoto model with uniform distribution of the natural frequencies $\omega_{i}$ but they are also valid for other frequency distributions. We found phase chaos for finite-dimensional approximations of the Gaussian distribution.

The Kuramoto model is probably the simplest network of oscillators in which phase chaos emerges and that is accessible for analytical treatment. However, our finding of phase chaos in coupled limit-cycle oscillators and coupled Rössler systems (Fig. 5), together with previous findings in other systems of coupled oscillators $[18,23,24]$ show that phase chaos is a common, probably universal phenomenon of networks of very different nature.

We thank P. Ashwin, A. Politi, A. Pikovsky, and M. Hasler for fruitful discussions. This study was supported by the Network of Excellence in Biosimulation (BioSim, Grant No. 005137).
[1] Y. Kuramoto, Chemical Oscillations, Waves and Turbulence (Springer-Verlag, Berlin, 1984).

[2] S. H. Strogatz, Physica D 143, 1 (2000); Nature (London) 410, 268 (2001); P. Ashwin and J. Borresen, Phys. Rev. E 70, 026203 (2004).

[3] K. Wiesenfeld, P. Colet, and S. H. Strogatz, Phys. Rev. Lett. 76, 404 (1996); Phys. Rev. E 57, 1563 (1998).

[4] G. Kozyreff, A. G. Vladimirov, and P. Mandel, Phys. Rev. Lett. 85, 3809 (2000).

[5] W. Wang, I. Z. Kiss, and J. L. Hudson, Chaos 10, 248 (2000); Phys. Rev. Lett. 86, 4954 (2001).

[6] C. S. Peskin, Mathematical Aspects of Heart Physiology (Courant Institute of Mathematical Sciences, New York, 1975).

[7] A. Nini A. et al., J. Neurophysiol. 74, 1800 (1995).

[8] P. A. Tass, Phase Resetting in Medicine and Biology (Springer, Berlin, 1999); Europhys. Lett. 53, 15 (2001); 55, 171 (2001); 59, 199 (2002); Biol. Cybern. 87, 102 (2002); 89, 81 (2003); Phys. Rev. E 66, 036226 (2002).

[9] P. A. Tass et al. (unpublished).

[10] Yu. Maistrenko et al., Phys. Rev. Lett. 93, 084102 (2004).

[11] Phase chaos or phase turbulence is known to appear in the Ginzburg-Landau and Kuramoto-Sivashinsky equations $[12,13]$. Chaotic behavior is found in generic nonlinear systems on multidimensional tori $[14,15]$.

[12] P. Manneville, Liapounov Exponents for KuramotoSivashinsky Model, Lecture Notes in Physics Vol. 230
(Springer, Berlin, 1985), p. 319.

[13] I. S. Aranson and L. Kramer, Rev. Mod. Phys. 74, 99 (2002).

[14] D. Ruelle and F. Takens, Commun. Math. Phys. 20, 167 (1971); S. Newhouse, D. Ruelle, and D. Takens, ibid. 64, 35 (1979).

[15] C. Baesens, J. Guckenheimer, S. Kim, and R. S. MacKay, Physica D 49, 387 (1991).

[16] V. S. Afraimovich and L. P. Shilnikov, Am. Math. Soc. Transl. 149, 176 (1991).

[17] J. D. Farmer, E. Ott, and J. A. Yorke, Physica D 7, 153 (1983).

[18] Z. Liu, Y.-C. Lai, and M. A. Matias, Phys. Rev. E 67, 045203(R) (2003).

[19] A. Politi (private communication).

[20] A. T. Winfree, The Geometry of Biological Times (Springer, New York, 1980); J. T. Ariaratnam and S. H. Strogatz, Phys. Rev. Lett. 86, 4278 (2001).

[21] Y. L. Maistrenko, O. V. Popovych, and P. A. Tass, Desynchronization and Chaos in the Kuramoto Model, Lecture Notes in Physics Vol. 671 (Springer, Berlin, 2005).

[22] M. G. Rosenblum, A. S. Pikovsky, and J. Kurths, Phys. Rev. Lett. 76, 1804 (1996).

[23] V. Hakim and W.-J. Rappel, Phys. Rev. A 46, R7347 (1992); N. Nakagawa and Y. Kuramoto, Prog. Theor. Phys. 89, 313 (1993); Physica D 75, 74 (1994); 80, 307 (1995).

[24] D. Topaj and A. Pikovsky, Physica D 170, 118 (2002). 